\title{
Use of Assisted Reproduction Techniques in Male Pathologies: Molecule Based Sperm Selection Methods
}

\begin{abstract}
Infertility is a widespread problem that is found in over $15 \%$ of couples. In $50 \%$ of the cases, the male factor is the key. In order to improve pregnancy rates in assisted reproductive techniques (ART), new methods to select sperm subpopulations are required. These methods are under constant evaluation, and their possible genetic and epigenetic effects on the offspring must be considered. New concepts such as DNA fragmentation and sperm apoptosis are being introduced in andrology and are considered to develop new techniques. Recently, molecule-based methods have been developed for the purpose of overcoming deficiencies in classical selection methods that involve sperm morphology and movement. One of these methods is physiologic intracytoplasmic sperm injection (PICSI), which selects spermatozoa through their property to bind to hyaluronic acid, a component of the cell-oocyte complexes that acts as a natural selector of spermatozoa. But the top rated method is based on the property of the protein annex in A5 (ANXA V) to bind to phosphatidylserine (PS), a marker of apoptosis. Magnetic-Activated Cell Sorting (MACS) conjugated with ANXA V (MACS-ANXA V) is used to remove apoptotic sperm with exposed PS from a semen sample. The use of MACS-ANX V seems to produce good quality sperm when it is evaluated by in vitro assays. But when MACS-ANXA V is used previous to ART, controversies persist regarding the benefits of sperm selection in reproductive outcomes. In this review, we consider the use of MACS-ANXA V, particularly in cases of male pathologies.
\end{abstract}

Keywords: Annexin V; Hyaluronic acid; DNA sperm fragmentation; Apoptosis; MACS

\author{
Mini Review \\ Volume 5 Issue 3 - 2017 \\ Marini Patricia Estela ${ }^{1,2}$, Munuce María \\ José ${ }^{2}$, Caille Adriana María ${ }^{2}$, Zumoffen \\ Carlos $^{2}$ and Teijeiro Juan Manuel ${ }^{1,3 *}$ \\ ${ }^{1}$ Instituto de Biología Molecular y Celular de Rosario, Consejo \\ de Investigaciones de la Universidad Nacional de Rosario, \\ Argentina \\ ${ }^{2}$ Laboratorio de Medicina Reproductiva, Facultad de Ciencias \\ Bioquímicas y Farmacéuticas, Universidad Nacional de \\ Rosario, Argentina \\ ${ }^{3}$ Concejo Nacional de Investigaciones Científicas y Técnicas, \\ Argentina \\ *Corresponding author: Juan Manuel Teijeiro, Laboratorio \\ de Medicina Reproductiva, Facultad de Ciencias Bioquímicas \\ y Farmacéuticas, Universidad Nacional de Rosario, \\ Argentina, Concejo Nacional de Investigaciones Científicas \\ y Técnicas, CONICET, Suipacha 531, S2002LRK, Rosario, \\ Santa Fe, Argentina, Tel: 54-341- 4350661 (ext 122); Email: \\ jteijeiro@fbioyf.unr.edu.ar
}

Received: June 09, 2017 | Published: August 17, 2017

\begin{abstract}
Abbreviations: WHO: World Health Organization; ROS: Reactive Oxygen Species; PS: Phosphatidylserine; EPS: Externalization of Phosphatidylserine; ART: Assisted Reproduction Techniques; IUI: Intra-Uterine Insemination; IVF: In Vitro Fertilization; ICSI: Intracytoplasmic Sperm Injection; PICSI: Physiologic Intracytoplasmic Sperm Injection; SU: Swim-Up; DG: Density Gradient; HA: Hyaluronic Acid; ANX V: annexin A5; MACS: Magnetic-Activated Cell Sorting; MACS-ANX V: Magnetic-Activated Cell Sorting coupled to ANX V; TESA: Testicular Sperm Aspiration
\end{abstract}

\section{Introduction}

Infertility is a widespread problem that refers to the inability to achieve a clinical pregnancy after a year or more of regular unprotected sexual intercourse [1]. It is found in over $15 \%$ of couples and $50 \%$ of the cases are due to male factor. Abnormal semen is responsible for $75 \%$ of all cases of male infertility. Semen quality is commonly assayed in andrology laboratories by standard analyses. These analyses should be performed according to the World Health Organization (WHO) guidelines [1] and involve the measures of volume, $\mathrm{pH}$, and sperm concentration, motility and morphology with strict criteria. As WHO guidelines semen analyses sometimes are not able to discriminate between fertile and infertile patients, and have a poor predictive value for the outcome of embryo development, the development of new techniques is required [2]. In recent years, the management of the male factor has undergone important changes with the introduction of novel concepts such as sperm DNA fragmentation and apoptosis. Therefore, new techniques are being developed in order to analyse these kinds of cell processes.

\section{Discussion}

Mammalian spermatozoa are highly polarized haploid cells that must suffer complex physiological changes before being able to fertilize an oocyte. These changes take place in vivo into the Fallopian tube and are collectively known as capacitation $[3,4]$. Some of the events inherent to capacitation are the production of reactive oxygen species (ROS) and membrane lipids scrambling, with the consequent externalization of phosphatidylserine (EPS) [5]. Apoptosis is a programmed cell death and it is generally accepted that EPS is a marker of apoptosis in most diploid cells. But this phenomenon is not well characterized in spermatozoa.

As mentioned before, sperm DNA fragmentation analysis is a novel concept introduced in the management of male infertility and it has been related to the presence of ROS. Irreversible DNA fragmentation may be produced during spermatogenesis, during sperm's journey through the male tract, by incorrect chromatin packaging, during apoptosis, or it may be caused in vitro by manipulation in laboratories. It is also enhanced by external factors such as environmental hazards, smoking and increased testicular temperature. Sperm DNA damage has also been related 
to pathologies such as cryptorchidism, varicocele, inflammatory processes, infections, cancer, chemotherapy, radiotherapy and stress [6]. Infertility is currently treated worldwide by assisted reproduction techniques (ART) which still show suboptimal efficiency. ART includes: intra-uterine insemination (IUI), in vitro fertilization (IVF) and intracytoplasmic sperm injection (ICSI), followed by embryo transfer. In IVF the gametes are placed together in artificial media for fertilization to occur. In ICSI instead, a spermatozoon is chosen and injected directly into the oocyte`s cytoplasm. Thus, in this last procedure spermatozoa bypass most physiological processes. As a result, sperm with low quality such as damaged DNA and other non-noticeable defects might fertilize an oocyte producing an embryo with developmental problems. Despite this fact, ICSI is the treatment of choice when a severe male factor is present.

In ART, an initial step is used to render sperm with higher fertilizing potential. This step is based on the premise that cervical mucus selectively allows only progressively motile sperm of normal shape to penetrate and migrate through the cervix [7]. This selection step is performed by conventional techniques such as glass wool filtration, swim-up (SU), and density gradient (DG) All these methods select a subpopulation of sperm with higher morphological and motility qualities. Among these spermatozoa, one spermatozoon is selected based only on its morphology and motility for ICSI. New methods are constantly being thought in order to optimize sperm selection for ICSI and recently biological and molecular approaches have been conveyed to this regard. Human oocytes are surrounded by hyaluronic acid (HA) that acts as a natural selector of spermatozoa. Based on this phenomenon a biological test using HA-coated slides has been developed [8]. In this method, spermatozoa that bind to HA-coated slides are selected and used for ICSI. The procedure is called "physiologic ICSI", PICSI [9]. Although some reports show that PICSI has a considerably higher probability $(\approx 5$ fold) to achieve pregnancy than ICSI, a meta-analysis of all available studies concluded that although an improvement in embryo quality is provided by PICSI over ICSI, the evidence is not enough to justify the routine use of PICSI [10].

Another method promotes the removal of sperm that have begun apoptosis from the sample. Sperm particularities such as scarce cytoplasm and organelles and transcriptional inactivity rise doubts about the similarities of its apoptosis with that of other cells. However, EPS is considered to occur in apoptotic sperm [11]. Thus, a novel method based on binding of the protein annexin A5 (ANX V) to EPS has been developed. In this method Magnetic-Activated Cell Sorting (MACS) is performed with colloidal superparamagnetic microbeads (50 $\mathrm{nm}$ diameter) coupled to ANX V (MACS-ANXA V). A diagrammatic description of different methods of sperm selection depicting the mechanism of action and anatomical structure of the human spermatozoa is shown in Figure 1. The sperm/micro-beads suspension is then loaded on a separation column, which is placed in a fitted magnet. Sperm bound to ANX V by EPS stay trapped in the matrix, and the separation process gives two sperm populations: EPS-negative (vital, non-apoptotic sperm with intact membranes) and EPSpositive (apoptotic cells) [12,13]. As it was mentioned before, sperm that have begun capacitation expose PS on their membrane surface, therefore, capacitated sperm may also be trapped into the column. MACS-ANX V was developed and commercialized by Miltenyi Biotec GmbH (Bergisch Gladbach, Germany) and is frequently used in combination with SU, DG or both.

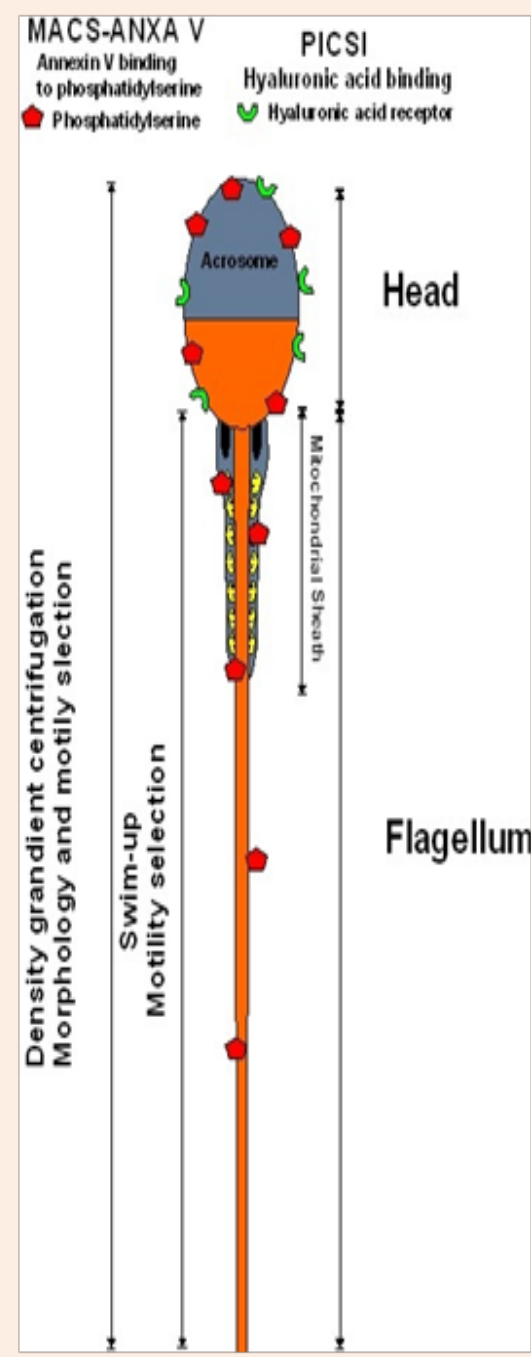

Figure 1: A diagrammatic description of different methods of sperm selection depicting the mechanism of action and anatomical structure of the human spermatozoa.

In vitro analysis of sperm populations obtained after MACSANXA V selection of semen from men exposed to environmental hazards as manganese and phthalates, results in a selected population with decreased apoptosis and DNA damage rates $[14,15]$. A study using DG-MACS-ANXA V, applied to semen from men with varicocele, showed no negative effects on sperm motility and isolation of a population of sperm with decreased DNA fragmentation rate [16]. Moreover, a study was performed to compare DG-MACS-ANXA V versus DG using samples from astheno teratozoospermic $(n=17)$ and teratozoospermic $(n=12)$ patients [17]. In this study a population of sperm with better chromatin quality was obtained after DG-MACS-ANXA V than upon DG alone. 
On the other hand, a randomized trial using donated oocytes and semen from unselected men, showed no differences in fertilization, implantation, pregnancy, and live-birth rates after selection of sperm with MACS-ANXA V compared with washed semen [18]. However, the born of a healthy baby was recorded using MACS-ANXA V for sperm selection from semen of a patient with bilateral varicocele, surgically treated, and with high rate of sperm DNA fragmentation [19]. Another study resulted in a single and a twin pregnancies after MACS-ANXA $V$ sperm selection from semen of a man with asthenoteratozoospermia and another man with surgically treated varicocele and high rate of sperm DNA fragmentation [20]. In other study that comprised oligozoospermic, asthenozoospermic, oligoasthenospermic and/ or teratozoospermic patients, improved sperm morphology and slightly higher implantation rates were noted in favour of MACSANX V over DG sperm selection [21]. According to a case report, MACS-ANXA V was used to select sperm obtained by testicular sperm aspiration (TESA) before ICSI in a man who had a traffic accident that resulted in paraplegia, without any chance of intercourse. In such case report, the born child was examined at the age of 4 finding normal development [22]. Another case report showed that the use of MACS-ANXA V on cryo-preserved spermatozoa with high DNA damage from a patient with nonHodgkin's lymphoma, resulted in the birth of healthy twins [23].

\section{Conclusion}

MACS-ANXA $\mathrm{V}$ is a promising new technology for sperm selection but its improvement in ART success is still under debate. Most of the studies described here have different experimental design, inclusion criteria and population size. To confirm or refute the use of this technique in clinical practice, further controlled and randomized studies are required. Moreover, oocytes coming from young women show a capability to repair sperm DNA damage. Thus, in order to test the efficiency of a new technique, standardized studies with the same oocytes quality (i.e. donated from women in programs of IVF), should be performed. While an improvement in the quality of sperm populations recovered by the new presented methods is evident by in vitro sperm analyses, the in vivo results need more, conclusive and evidence. It appears that when the damage in sperm DNA is not severe, oocytes of good quality can repair it and result in successful pregnancies, but when sperm DNA damage is severe, such as in patients with varicocele, cancer or men exposed to environmental hazards, MACS-ANXA V seems to be an option to obtain clinical pregnancies and healthy born children. Regarding apoptosis, taking into account that such phenomenon begins in the testicles it would be important to consider the use of MACS-ANXA V in combination with TESA.

\section{Acknowledgement}

We thank Virginia Castro for English assistance.

\section{References}

1. World Health Organization (2010) WHO laboratory manual for the examination and processing of human semen. ( $5^{\text {th }}$ edn) Geneva, Switzerland: WHO.
2. Chapuis A, Gala A, Ferrières-Hoa A, Mullet T, Bringer-Deutsch S, et al. (2017) Sperm quality and paternal age: effect on blastocyst formation and pregnancy rates. Basic Clin Androl 27: 2-9.

3. Austin C (1952) The capacitation of the mammalian sperm. Nature 170(4321): 326.

4. Chang M (1952) Fertilizing capacityof spermatozoa deposited into the Fallopian tubes. Nature 168(4277): 697-698.

5. Tosti E, Ménézo Y (2016) Gamete activation: basic knowledge and clinical applications. Hum Reprod Update 22(4): 420-439.

6. Quintero-Vásquez A, Castillo-Cadena J (2015) Male infertility and spermatic DNA fragmentation: A current problema. TIP Revista Especializada en Ciencias Químico-Biológicas, 18(2): 144-151.

7. Yanagimachi R (1994) Mammalian fertilization: The physiology of reproduction. E Knobil and J Neill (Eds.), Raven Press, New York, USA.

8. Huszar G, Jakab A, Sakkas D, Ozenci CC, Cayli S, et al. (2007) Fertility testing and ICSI sperm selection by hyaluronic acid binding: clinical and genetic aspects. Reprod Biomed Online 14(5): 650-663.

9. Erberelli RF, Salgado RM, Pereira DH, Wolff P (2017) Hyaluronanbinding system for sperm selection enhances pregnancy rates in ICSI cycles associated with male factor infertility. JBRA Assist Reprod 21(1): 2-6.

10. Beck-Fruchter R, Shalev E, Weiss A (2016) Clinical benefit using sperm hyaluronic acid binding technique in ICSI cycles: a systematic review and meta-analysis. Reprod Biomed Online 32(3): 286-298.

11. You L, Wang YX, Zeng Q Li M, Huang YH, et al. (2015) Semen phthalate metabolites, spermatozoa apoptosis, and DNA damage: a cross-sectional study in China. Environ Sci Technol 49(6): 38053812.

12. Grunewald S, Paasch U, Glander HJ (2001) Enrichment of non-apoptotic human spermatozoa after cryopreservation by immunomagnetic cell sorting. Cell Tissue Bank 2(3): 127-133.

13. Grunewald S, Paasch U (2013) Methods in molecular biology series. Sperm preparation and selection techniques: sperm selection for ICSI using Annexin VJM Walker, Springer, New York Heidelberg Dordrecht London, pp. 257-262.

14. Wang YX, Sun Y, Huang Z, Wang P, Feng W, et al. (2016) Associations of urinary metal levels with serum hormones,spermatozoa apoptosis and sperm DNA damage in a Chinese population. Environ Int 94: 177-188.

15. Wang YX, Zeng Q, Sun Y, You L, Wang P, et al. (2016) Phthalate exposure in association with serum hormone levels, sperm DNA damage and spermatozoa apoptosis: A cross-sectional study in China. Environ Res 150: 557-565.

16. Degheidy T, Abdelfattah H, Seif A, Albuz FK, Gazi S, et al. (2015) Magnetic activated cell sorting: an effective method for reduction of sperm DNA fragmentation in varicocele men prior to assisted reproductive techniques. Andrologia 47(8): 892-896.

17. Delbes G, Herrero MB, Troeung ET, Chan PT (2013) The use of complimentary assays to evaluate the enrichment of human sperm quality in asthenoteratozoospermic and teratozoospermic samples processed with Annexin-V magnetic activated cell sorting. Andrology 1(5): 698-706. 
18. Romany L, Garrido N, Motato Y, Aparicio B, Remohí J, et al. (2014) Removal of annexin V-positive sperm cells for intracytoplasmic sperm injection in ovum donation cycles does not improve reproductive outcome: a controlled and randomized trial in unselected males. Fertil Steril 102(6): 1567-1575.

19. Rawe VY, Boudri HU, Alvarez Sedo C, Carro M, Papier S, et al. (2010) Healthy baby born after reduction of sperm DNA fragmentation using cell sorting before ICSI. Reprod Biomed Online 20(3): 320323

20. Polakde FE, Denaday F (2010) Single and twin ongoing pregnancies in two cases of previous ART failure after ICSI performed with sperm sorted using annexin V microbeads. Fertil Steril 94(1): 351. e15-18.
21. Dirican EK, Ozgün OD, Akarsu S, Akin KO, Ercan O, et al. (2008) Clinical outcome of magnetic activated cell sorting of nonapoptotic spermatozoa before density gradient centrifugation for assisted reproduction. J Assist Reprod Genet 25(8): 375-381.

22. Lukaszuk K, Wcislo M, Liss J, Stachowicz A, Jakiel G, et al. (2015) First pregnancy, somatic and psychological status of a 4-year-old child born following Annexin V TESA sperm separation. AJP Rep 5(2): 105-108.

23. Herrero MB, Delbes G, Chung JT, Son WY, Holzer H, et al. (2013) Case report: the use of annexin $V$ coupled with magnetic activated cell sorting in cryopreserved spermatozoa from a male cancer survivor: healthy twin newborns after two previous ICSI failures. J Assist Reprod Genet 30(11): 1415-1419. 